\title{
Mechanisms underlying the activation of TERT transcription and telomerase activity in human cancer: old actors and new players
}

\author{
Xiaotian Yuan ${ }^{1,2} \cdot$ Catharina Larsson ${ }^{3} \cdot$ Dawei $\mathrm{Xu}^{2}$ \\ Received: 19 May 2019 / Revised: 20 June 2019 / Accepted: 20 June 2019 / Published online: 8 July 2019 \\ (c) The Author(s) 2019. This article is published with open access
}

\begin{abstract}
Long-lived species Homo sapiens have evolved robust protection mechanisms against cancer by repressing telomerase and maintaining short telomeres, thereby delaying the onset of the majority of cancer types until post-reproductive age. Indeed, telomerase is silent in most differentiated human cells, predominantly due to the transcriptional repression of its catalytic component telomerase reverse transcriptase (TERT) gene. The lack of telomerase/TERT expression leads to progressive telomere erosion in dividing human cells, whereas critically shortened telomere length induces a permanent growth arrest stage named replicative senescence. TERT/telomerase activation has been experimentally shown to be essential to cellular immortalization and malignant transformation by stabilizing telomere length and erasing the senescence barrier. Consistently, TERT expression/telomerase activity is detectable in up to $90 \%$ of human primary cancers. Compelling evidence has also accumulated that TERT contributes to cancer development and progression via multiple activities beyond its canonical telomere-lengthening function. Given these key roles of telomerase and TERT in oncogenesis, great efforts have been made to decipher mechanisms underlying telomerase activation and TERT induction. In the last two decades since the TERT gene and promoter were cloned, the derepression of the TERT gene has been shown to be achieved typically at a transcriptional level through dysregulation of oncogenic factors or signaling, post-transcriptional/translational regulation and genomic amplification. However, advances in high-throughput next-generation sequencing technologies have prompted a revolution in cancer genomics, which leads to the recent discovery that genomic alterations take center stage in activating the $T E R T$ gene. In this review article, we summarize critical mechanisms activating TERT transcription, with special emphases on the contribution of TERT promoter mutations and structural alterations at the TERT locus, and briefly discuss the underlying implications of these genomic events-driven TERT hyperactivity in cancer initiation/progression and potential clinical applications as well.
\end{abstract}

\section{Introduction}

Cancer formation/progression results from the accumulation of genetic mutations in cells [1]. Because every cell is exposed to mutagens with each round of cell division to a

Xiaotian Yuan

Xiaotian.Yan@ki.se

$\triangle$ Dawei Xu

Dawei.Xu@ki.se

1 School of Medicine, Shandong University, 250012 Jinan, People's Republic of China

2 Department of Medicine, Center for Molecular Medicine (CMM) and Bioclinicum, Karolinska Institutet and Karolinska University Hospital Solna, 17164 Solna, Sweden

3 Department of Oncology-Pathology and Bioclinicum, Karolinska Institutet and Karolinska University Hospital Solna, 17164 Solna, Sweden similar extent, and larger animals undergo more cell divisions and experience more mutagenic exposure, the risk of developing cancer should theoretically be higher in species with bigger body sizes and longer lifespan [2]. However, despite 1000 -fold more cells and/>30-fold longer lifespan in the human than in the mouse, human cancer risk is actually much lower: Approximately one-quarter of aged people die of cancer, whereas this number can reach up to $90 \%$ in aged mice [2]. Several lines of evidence have suggested that naturally occurring strategies against cancer have evolved in long-lived and large-bodied mammalian species, thereby delaying the onset of cancer until post-reproductive age [2]. 
For instance, large mammalian species including humans (with body mass $>5-10 \mathrm{~kg}$ ) acquire a strong cancer-protection means by repressing telomerase, a ribonucleoprotein enzyme that catalyzes telomeric DNA lengthening on chromosome termini [2, 3]. In contrast, mouse somatic cells are TERT/ telomerase-proficient. Moreover, most laboratory mice carry 5-10-fold longer telomere than do humans [3]. Telomerase repression coupled with short telomeres in human cells is believed to confer them a potent barrier to transformation. In support of this view, Hahn et al. [4] experimentally demonstrated that two oncogenes (the simian virus 40 large-T oncoprotein and an oncogenic allele of H-ras) in combination with telomerase activation through ectopic TERT expression are required to directly convert human epithelial cells and fibroblasts into malignant cells, whereas these two oncogenes are sufficient to transform rodent cells into tumorigenic cells without introducing ectopic TERT.

Mechanistically, this telomerase repression and/or shorter telomeres in human cells function to prevent uncontrolled cellular proliferation [2]. The lack of telomerase leads to progressive telomere erosion in dividing human cells due to the intrinsic feature of DNA polymerase [5]. When telomere length shortens to a critical size and telomeres become dysfunctional, the DNA damage response pathway is activated and cells are triggered to enter into a permanent growth arrest stage named replicative senescence. Senescence is believed to act as a very effective barrier against cancer by blocking proliferation and genetic mutations resulting from DNA replication. As infinite proliferation is a hallmark of malignant cells [6], conceivably, overcoming the senescence barrier by telomere stabilization is required in oncogenesis, and, in most cases, this is achieved via telomerase activation $[5,6]$.

Telomerase is a multi-unit complex, but its core enzyme is only composed of a catalytic component TERT and internal telomerase RNA template (TERC) [5]. TERC is ubiquitously expressed in various human cells while the $T E R T$ gene is stringently repressed in most human somatic cells, which consequently results in telomerase silencing. Thus, TERT is a rate-limiting determinant for controlling telomerase activity. Indeed, numerous studies have unequivocally demonstrated that TERT induction is required for human cells to acquire telomerase activity. Bodnar et al. [7] elegantly showed that the ectopic introduction of TERT into telomerase-negative human fibroblasts induces telomerase activity, thereby leading to telomere lengthening and immortalization.

TERT induction/telomerase activation confers unlimited proliferation potential to cancer cells by stabilizing their telomere length, while recent observations reveal its multiple oncogenic activities independently of a telomerelengthening function, which include its effect on mitochondrial and ubiquitin-proteasomal function, DNA damage repair, gene transcription, microRNA expression, etc. [8-16]. Importantly, TERT was previously shown to promote the proliferation of normal mouse stem cells by recruiting chromatin-remodeling factor $\operatorname{Brg} 1$ to $\beta$-catenin target genes for their transcriptional activation [17]; and more recently, TERT was found to directly interact with $\beta$-catenin and robustly amplify its transcriptional outputs, thereby stimulating epithelial mesenchymal transformation (EMT) and stemness of cancer cells $[12,13]$. TERT is also required for symmetric stem cell division and its high expression significantly increases cancer stem cell (CSC) pool and self-renewal in prostate cancer [13]. It has also been revealed that TERT displays a RNA-dependent RNA polymerase (RdRP) activity, thereby regulating mitotic progression and cancer stem cell traits [18]. In addition,

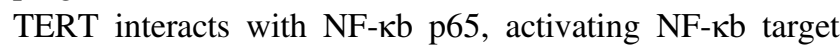
genes and upregulating the expression of a number of metalloproteinases (MMPs) in cancer cells [14]. The enhanced EMT, stemness, and MMP expression all contribute to invasiveness and metastasis in cancer, which indicates an important role of TERT in cancer progression. Liu et al. further demonstrated that TERT served as a partner for the transcription factor $\mathrm{Sp} 1$ to facilitate cancer angiogenesis [19]. We recently found that this same mechanism was involved in TERT-mediated aberrant DNA methylation and silencing of tumor suppressor genes in hepatocellular cell carcinoma (HCC) cells [20]. Taken together, TERT or telomerase may contribute to multicancer hallmarks via its telomere lengthening-dependent and independent functions (Fig. 1).

The evolutionarily acquired telomerase repression as a protective strategy against cancer, together with the multiple oncogenic effects of TERT/telomerase as revealed by modern experimental approaches, have pointed to the importance of TERT/telomerase in cancer development and progression. Therefore, great efforts have been made to dissect how the TERT gene is transcriptionally de-repressed and telomerase is activated during oncogenesis. Here, we present an overview of the mechanistic insights into cancerspecific TERT expression and biological/clinical implications, paying special attentions to the contribution of genomic aberrations to the TERT trans-activation.

\section{The TERT gene, and its promoter and transcripts}

The TERT gene consists of 16 exons and 15 introns within a $\sim 40 \mathrm{~kb}$ gene body and is localized on the short arm of the chromosome 5 (5p.15:33), a megabase distance from the $5 \mathrm{p}$ end (Fig. 2) [21, 22]. The TERT harbors a single promoter embedded in a $\mathrm{CpG}$ island $(-1800$ to +2300 relative to ATG), while the proximal core promoter is within a $330 \mathrm{bp}$ upstream and $37 \mathrm{bp}$ downstream from ATG $(-330$ to +37$)$ [23-26]. Remarkably, the TERT promoter region is rich with 


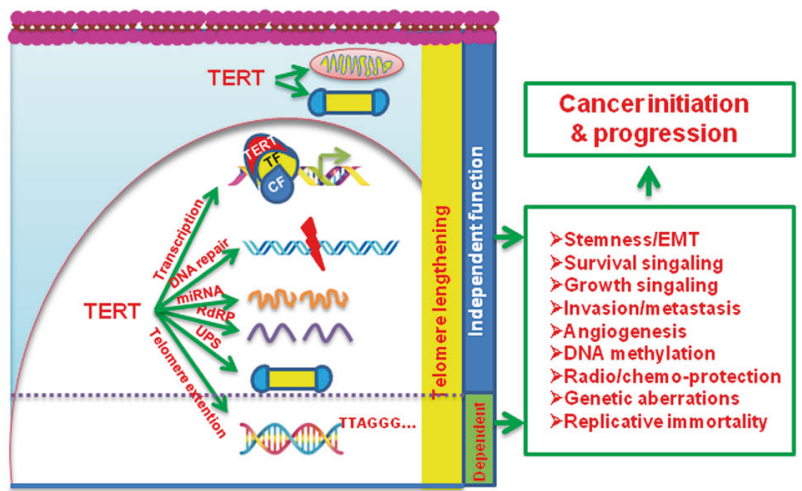

Fig. 1 Multiple oncogeneic roles for TERT in cancer development and progression. TERT/telomerase activation is required for transformation of human cells and infinite proliferation by stabilizing telomere length (Telomere lengthening-dependent). The telomere lengtheningindependent functions of TERT significantly contribute to cancer initiation/progression, which include its effects on mitochondria, ubiquitin-proteasomal system (UPS), gene transcription, microRNA (miRNA) expression, DNA damage repair, RNA-dependent RNA polymerase (RdRP) activity. CF co-factor; EMT epithelialmesenchymal transition, TF transcription factor. The effect of TERT on UPS predominantly occurs in the nucleus, but is also possible in the cytoplasma

biding motifs for multiple transcription factors including the MYC oncogene (E-box) and Sp1 (GC box), but lack TATA and CAAT boxes $[21,27]$. On the other hand, the promoter also carries sites for repressor binding. Another unique feature is that the TERT promoter is unmethylated in normal human cells, whereas methylated in malignant cells, and Lee et al. identified the 52 CpG-containing TERT hypermethylated oncological region (THOR) as a cancer-associated epigenetic mechanism of TERT upregulation [23-26]. Several lines of evidence suggest that the unmethylated promoter sequence favors a repressor-binding [23].

The TERT is a single copy gene with a single transcription start site, but subject to alternatively splicing regulation [21, 28]. More than 20 splicing variants have been identified, while the full-length TERT mRNA is the only one that translates into a functional protein for telomerase activity [28]. Some of these spliced variants such as $\alpha$-variants, $\beta$-variants, and $\gamma$-variants miss parts of sequences encoding the reverse transcriptase domain, and may therefore exert a dominant negative effect [28]. Intriguingly, certain normal human cells have been observed to express non-functional transcripts [28-30], but the underlying physiological significance remains unclear.

\section{Aberrant TERT transcription in human cancer: dysregulated positive and/or negative players}

The cloning of the TERT promoter and identification of its binding motifs for various transcription factors has contributed to profound insights into TERT/telomerase

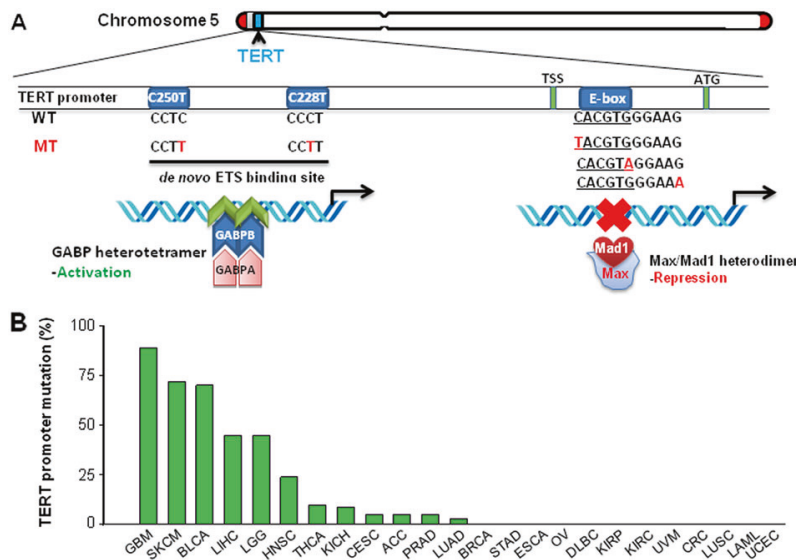

Fig. 2 TERT promoter mutations in human cancer. a Schematic presentation of TERT promoter mutations and relevant transcription factors. The TERT gene at chromosome $5 \mathrm{p}$ and its promoter is shown. $\mathrm{C}>\mathrm{T}$ mutation occurs at one of both positions of the TERT proximal promoter $(-124$ and -146 to ATG for C228T and C250T, respectively) in malignant cells, which create de novo ETS binding motifs. The ETS family members GABPA and GABPB form heterotetramers that bind to the de novo ETS site and activate TERT transcription. The E-box (CACGTG) sequence mutation was recently identified in clear cell renal cell carcinoma (ccRCC), which may lead to the dissociation of the repressor MAX/Mad1 complex from E-box, thereby derepressing the TERT gene. b The frequency of TERT promoter mutations in a panel cancer types from the TCGA dataset analyses [49]. GBM glioblastoma multiforme, SKCM skin cutaneous melanoma, BLCA bladder urothelial carcinoma, LIHC liver hepatocellular carcinoma, LGG brain lower-grade glioma, HNSC head and neck squamous cell carcinoma, THCA thyroid carcinoma, KICH kidney chromophobe, CESC cervical squamous cell carcinoma and endocervical adenocarcinoma, ACC adrenocortical carcinoma, PRAD prostate adenocarcinoma, LUAD lung adenocarcinoma, BRCA breast invasive carcinoma, STAD stomach adenocarcinoma, ESCA esophageal carcinoma, OV ovarian serous cystadenocarcinoma, DLBC lymphoid neoplasm diffuse large B cell lymphoma, KIRP kidney renal papillary cell carcinoma, KIRC kidney renal clear cell carcinoma, UVM Uveal melanoma, SARC sarcoma, CRC colorectal carcinoma, LAML acute myeloid leukemia, LUSC lung squamous cell carcinoma, UCEC uterine corpus endometrial carcinoma

regulation in human cells [21,27]. The early studies showed that TERT promoter activity correlated closely with TERT mRNA expression: significantly higher in telomerasepositive cancer cells than in telomerase-negative normal ones, which suggests that controlling TERT expression at a transcriptional level represents a fundamental mechanism to activate telomerase in cancer cells [21,27].

The findings accumulated in the last 20 years have shown that the transcriptional regulation of the TERT gene occurs at multiple levels by various positive and negative factors or signaling pathways. The TERT promoter is bound by different transcription factors, responds to numerous signals and integrates these diverse and dynamic inputs to set the TERT expression output [31]. Furthermore, epigenetic effects on chromatin structure and remodeling of the TERT promoter region add another layer controlling the 
TERT transcription [31]. In addition, many factors indirectly regulate TERT transcription by cooperating with transcription factors or other regulatory elements in a contextdependent or independent manner. All these regulators coordinately and tightly control the TERT gene to ensure its silence in most normal cells, while its expression at the right time, right place and right quantity only in a small subset of cells, such as activated lymphocytes and stem/progenitor cells [31]. However, this tightly regulated balance is readily disrupted in malignant cells, most likely due to aberrant expression of positive regulators or silencing/sequestering of negative ones [31]. The most typical example are the Myc/Max/Mad1 network proteins [31-33]. MYC is the first identified cellular oncogene to activate telomerase [34]. Ectopic expression of c-MYC in human fibroblasts or epithelial cells robustly induced TERT expression and telomerase activity [34]. In leukemic HL60 cells, a high level of c-Myc expression is coupled with its binding to the E-Boxes on the TERT proximal promoter and TERT mRNA abundance. Once HL60 cells are induced to undergo terminal differentiation, c-Myc expression is diminished, whereas Mad1 levels increase and it consequently replace c-MYC on the TERT promoter, repressing TERT transcription [32]. Casillas et al. determined the TERT gene trans-activation by endogenous c-Myc during the transformation process of human fibroblasts, and they observed that the endogenous c-Myc expression resulted in a switch from Mad1/Max to cMyc/Max binding to E-boxes on the TERT promoter, TERT expression and telomerase activation [33]. These changes at the TERT promoter are totally opposite to what happens in differentiated leukemic HL60 cells [32]. In addition, many other factors regulate TERT transcription through the Myc/Max/Mad protein family or different mechanisms. These regulators include the TGF- $\beta / \mathrm{Smad}$ signaling pathway, endoplasmic reticulum stress, NFX1 Tax, estrogen, Ets, DJ-1, E2F, survivin, HIFs, FoxM1, Reptin, Wnt/ $\beta$-Catenin, Arsenic, Aurora-A, cold inducible RNA-binding protein, various growth factors, and cytokines, etc. [31, 35-44].

The presence of transcription factors is essential to the TERT transcription regulation. However, gene transcription involves not only the assembly of transcription factors at promoter/ enhancer regions, but also the regulation of accessibility to DNA, a process controlled by an epigenetic mechanism [31, 32, 42, 45]. Thus, epigenetic factors are another group of proteins that modulate TERT transcription. DNA methylation, histone acetylation, methylation, and phosphorylation have all been shown to be involved in the TERT transcription regulation [31, 32, 42, 45, 46]. The TERT promoter is in general unmethylated in normal cells, while its methylation is required for TERT expression and telomerase activation in cancer cells [23-26]. Histone acetylation/deacetylation was shown to be a common underlying feature to TERT transactivation/repression in human cells [31, 32, 42, 45]. Mechanistically, transcription factors Myc/Max/Mad and Sp1 interact with and recruit histone acetyltransferases (HATs) or histone deacetylases (HDACs) to the TERT promoter, dependent on the promoter status and cellular contexts [31, 32, 42]. In addition, SMYD3 as a histone methytransferase is capable of directly binding to CCCTCC sequences on the TERT promoter and specifically catalyzes $\mathrm{H} 3-\mathrm{K} 4$ tri-methylation, through which TERT transcription is activated. SMYD3-mediated H3-K4 tri-methylation is required for optimal occupancy of c-MYC and Sp1 on the TERT promoter [45]. Thus, epigenetic factors intimately crosstalk and cooperate with transcription factors to exert their regulatory effects on TERT transcription.

In addition to the endogenous TERT regulators discussed above, certain tumor viruses encode proteins that stimulate TERT transcription. These exogenous regulators include Epstein-Barr virus (EBV), cytomegalovirus (CMV), Kaposi sarcoma-associated herpesvirus (KSHV), human papillomavirus (HPV), hepatitis $\mathrm{B}$ virus (HBV), hepatitis $\mathrm{C}$ virus (HCV), human T-cell leukemia virus-1 (HTLV-1), and others $[47,48]$. The HPV E6 is the most extensively studied viral oncoprotein for its role in the TERT transcription. E6 forms a tertiary complex with E6AP and c-Myc, and such complex then binds to the E-box in the TERT core promoter and subsequently induces promoter activation [47]. The CMV early protein 72 robustly activates $T E R T$ transcription by interacting with $\mathrm{Sp} 1$ [48]. Thus, the targeted activation of TERT transcription is one of the key mechanisms for virus-mediated carcinogenesis.

\section{Aberrant TERT transcription in human cancer: genomic alterations as new players at center stage}

Advances in high-throughput sequencing technologies have enabled comprehensive genomic characterization of various human malignancies and numerous genomic aberrations have been unraveled. Barthel et al. [49] recently examined telomere length, TERT expression and related genomic alterations in 31 cancer types derived from The Cancer Genome Atlas (TCGA) cohort of patients, and they found that the TERT promoter mutations, and focal amplification/ rearrangements were intimately associated with acquisition of TERT expression and telomerase activity in tumors (Figs. 2 and 3). These analysis results, together with recent findings by others, suggest that genomic aberrations act as pivotal players in the activation of the TERT transcription.

\section{The recurrent TERT promoter mutations in human cancer: gain-of-function}

TERT promoter mutations occur predominantly at two hotspots of chromosome $5(1,295,228$ and $1,295,250$, or -124 and $-146 \mathrm{bp}$ from the ATG) with a cytidine-to-thymidine 

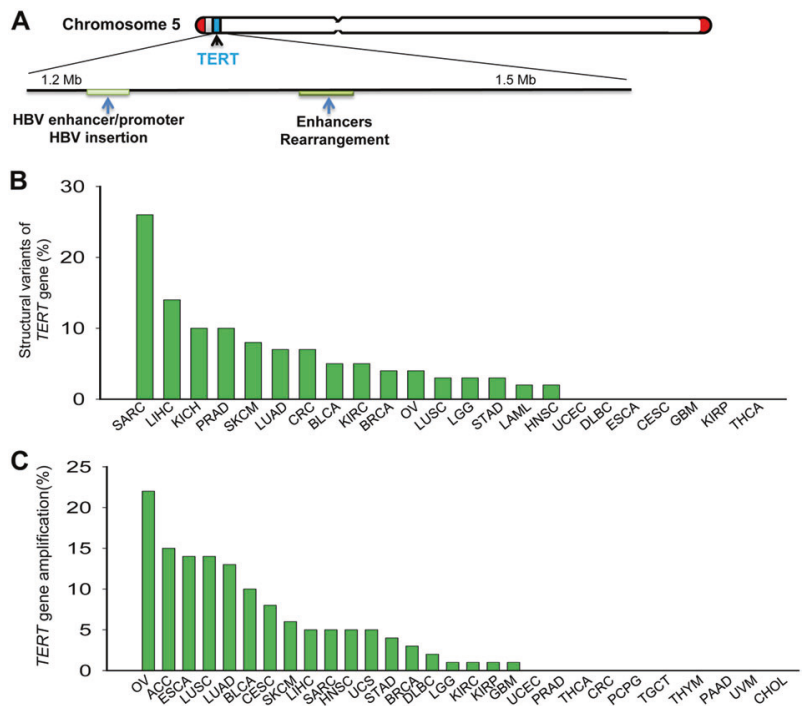

Fig. 3 The structural alterations and amplification of the TERT gene in human cancer. a Schematic presentation of rearrangements and oncoviral insertation of the TERT locus. Left: The rearrangements occur most frequently in a $50 \mathrm{~kb}$ region proximal of the TERT gene, although the translocation to other chromsomes is also observed. The rearrangements are not random events, which often juxtapose the TERT coding sequence to strong enhancer elements. Right: Most integration breakpoints for onco-viral insertions are located in the TERT promoter region, and almost all the integrations contained at least one viral gene enhancer or promoter. $\mathbf{b}$ and $\mathbf{c}$ The structural variants and amplification of the TERT gene in a panel of human malignancies from the TCGA cohort analyses, respectively. GBM glioblastoma multiforme, SKCM skin cutaneous melanoma, BLCA bladder urothelial carcinoma, LIHC liver hepatocellular carcinoma, LGG brain lower-grade glioma, HNSC head and neck squamous cell carcinoma, THCA thyroid carcinoma, CESC cervical squamous cell carcinoma and endocervical adenocarcinoma, ACC adrenocortical carcinoma, PRAD prostate adenocarcinoma, LUSC lung squamous cell carcinoma, LUAD lung adenocarcinoma, PCPG Pheochromocytoma and paraganglioma, LUSC lung squamous cell carcinoma, TGCT testicular germ cell tumor, BRCA breast invasive carcinoma, STAD stomach adenocarcinoma, ESCA esophageal carcinoma, OV ovarian serous cystadenocarcinoma, DLBC lymphoid neoplasm diffuse large B cell lymphoma, KIRP kidney renal papillary cell carcinoma, KIRC kidney renal clear cell carcinoma, KICH kidney chromophobe, UVM uveal melanoma, SARC sarcoma, THYM thymus, CRC colorectal carcinoma, LAML acute myeloid leukemia, UCEC uterine corpus endometrial carcinoma, $\mathrm{CHOL}$ cholangiocarcinoma

$(\mathrm{C}>\mathrm{T})$ dipyrimidine transition, thus named $\mathrm{C} 228 \mathrm{~T}$ and C250T, respectively (Fig. 2a). These mutations, originally identified in sporadic and familiar malignant melanomas in 2013 [50, 51], have since then been widely investigated and observed in various types of human cancer with different frequencies (summarized in details in recent reviews [31, 52]). Glioblastoma, malignant melanoma, urothelial bladder cancer, myxoid liposarcoma, and certain subtypes of skin cancer and medulloblastoma exhibit the highest TERT promoter mutation rate (up to $80-90 \%$ ). The intermediate level of the mutation frequency $(15-50 \%)$ is observed in thyroid cancer, hepatocellular carcinoma (HCC), upper tract urinary carcinoma (UTUC), head and neck cancer, ovarian clear cell carcinoma and among others (Fig. 2). Whereas the rest of examined tumor types (lung, breast, gastrointestinal, prostate and kidney cancer, and almost all hematological malignancies) lack the mutations or have a low mutation rate $(<10 \%)$. An average of $27 \%$ tumors was observed to bear TERT promoter mutations in the TCGA pooled cohort, and they have so far been the most common mutations identified in non-coding regulatory regions in human cancer [49].

The C228T mutation is more prevalent than C250T among various malignancies and their presence is mutually exclusive, which suggests their functional redundancy. Indeed, primary tumors bearing either mutation tend to express higher levels of TERT mRNA and telomerase activity, implying a stimulatory effect on TERT expression $[31,49]$. Creating a C228T mutation in the TERT promoter region using a CRISPR technique in human pluripotent stem cells, Chiba et al. found that these cells constitutively expressed TERT and telomerase even after having undergone terminal differentiation, in sharp contrast to the wildtype (wt) TERT promoter-bearing stem cell-derived progenies where the TERT transcription was shut down following cellular differentiation [53]. Moreover, the differentiated cells with mutant TERT promoter carried longer telomeres and erased replicative senescence imposed by telomere attrition as seen in normal cells [53]. Similarly, $\mathrm{Li}$ et al. introduced the $\mathrm{C} 228 \mathrm{~T}$ mutation into the TERT promoter in normal human bladder stem cells and this single event was sufficient to drive transformation of these stem cells [54]. Thus, their findings provide direct evidence that the presence of $\mathrm{C} 228 \mathrm{~T}$ or C250T mutation confers cells immortality or sustained proliferation potential and promotes their transformation by activating telomerase.

To determine how these two mutations upregulate TERT expression, Huang et al. [51] generated C228T-bearing or C250T-bearing TERT promoter reporter constructs and observed a robust increase of the promoter activity, suggesting a gain-of-function effect on the TERT transcription. It has now been clear that these mutations create a de novo binding site for ETS transcription factors [51, 55]. Bell et al. and Mancini et al. further showed that GA-binding proteins (GABPA and GABPB1), the members of the ETS family transcription factors, were specifically recruited to the mutant rather than wt TERT promoter in cancer cells, thereby activating TERT transcription and telomerase (Fig. 2) $[55,56]$. Consistently, inhibiting the expression of GABPA or GABPB1 rather than other ETS members led to diminished TERT expression in cancer cells bearing a mutant TERT promoter $[55,56]$. In cancer cells carrying heterozygous TERT promoter mutations, the mutant promoter recruits GABPA and exhibits the H3K4me2/3 mark of active chromatin [57]. In contrast, the wild-type allele 
retains the H3K27me3 mark of epigenetic silencing [57]. These results suggest that only the mutant promoters are transcriptionally active. Moreover, GABPA needs to form a heterotetramer complex $\left[(\mathrm{GABPA} / \mathrm{GABPB})_{2}\right]$ with its partner GABPB1 or GABPB2 through which DNA binding and transcriptional regulation is achieved. Mancini et al. showed that mutant TERT promoter-harboring glioblastoma cells similarly exhibited decreased TERT expression upon GABPB1 knockdown, and these GABPB1-depleted cells also suffered impaired proliferation/survival, telomere shortening/dysfunction and attenuated tumorigenic ability [56]. Given these findings, together with previously observed oncogenic effects on prostate, breast cancer and leukemia [58], GABPA/B1 has recently been suggested as a novel therapeutic target for tumors harboring a mutant TERT promoter [56].

Surprisingly, however, our results obtained from thyroid cancer (TC) studies question it as a therapeutic target [59]. First, GABPA depletion leads to significant downregulation of TERT expression in TC-derived cells independently of TERT promoter mutations; Second, GABPA expression is negatively correlated with TERT expression in primary tumors from TC patients; Third, GABPA depletion robustly increases thyroid cancer cell invasion despite a diminished TERT expression. Mechanistically, we identified DICERI, a component of the microRNA machinery to inhibit cancer metastasis, as a direct target gene for GABPA. By promoting DICER1 expression, GABPA inhibits the invasive phenotype of thyroid cancer cells. Forth, lower rather than higher GABPA expression is positively correlated with aggressive disease and poor outcomes in TC patients. Finally, knocking-down GABPB1 expression in TC cells similarly facilitated invasiveness while inhibited TERT expression (Unpublished data). These observations raise a number of critical questions: How does GABPA regulate TERT expression in wt TERT promoter-carrying TC cells? Whether the effects of GABPA on the wt TERT promoter and cancer development/progression are context-dependent? Whether GABPA could serve as a therapeutic target for cancer? Elucidating these issues should be both biologically and clinically important.

In addition to $\mathrm{C} 228 \mathrm{~T}$ and $\mathrm{C} 250 \mathrm{~T}$ mutations, $\mathrm{CC}>\mathrm{TT}$ tandem mutations at position $-124 /-125 \mathrm{bp}$ and $-138 /-139$ (from ATG) were found in a subset of cancer [31, 60]. These two tandem mutations also lead to the creation of the ETS transcription factor-binding motif [31]. More recently, the mutation at the MYC binding site in the TERT promoter was documented in $8 \%$ of tumors from patients with clear cell renal cell carcinoma (ccRCC) (Fig. 2a) [61]. It is suggested that this type of the mutation may prevent the binding by repressors in the MYC network family, thereby de-repressing the TERT transcription [61]. Tumors carrying these mutations have the longest telomere compared to those with wt and C228T/C250T-TERT promoters [61]. However, the exact functional implication of this genetic alteration is unclear, and it is also unknown if this mutation is unique to ccRCC or present in other cancers, which calls for further investigations.

The TERT promoter mutation rate varies significantly from undetectable to more than $90 \%$ among studied human malignancies, and it remains poorly understood what cause such differential mutation distributions among different types of cancer [31]. We as well as others found that the presence of TERT promoter mutations was negatively correlated with telomere length in tumors [31, 62]. Moreover, senior age is also intimately associated with the mutation occurrence [62]; because progressive telomere erosion occurs with increased age, this observed agemutation link is likely due to shorter telomeres. It is well established that dysfunctional telomeres trigger genomic instability [5, 63], and conceivably, shortened telomeres may be a key driving-force for the onset of the TERT promoter mutation during malignant transformation. This view is strongly supported by a recent report from Garcia's group. They observed that the spontaneous acquisition of TERT promoter mutations was selected for in blood cells from non-tumor individuals with very short telomere due to germline defects in the TERT protein or other telomerase components [64]. We found that the germline variants at the TERT locus significantly affected the TERT promoter mutation rate in HCC patients, and the TERT rs2736100-CC cancer risk genotype was more frequently seen in patients with a wt TERT promoter [65]. This variant is known to upregulate TERT expression, thereby counteracting telomere shortening [66].

\section{TERT rearrangements and onco-viral DNA insertions: connecting to enhancer hijacking}

The cancer genomic landscape mapping has recently identified both rearrangements and oncogenic viral genome insertions at the TERT locus as novel mechanisms to upregulate TERT expression by hijacking enhancers (Fig. 3a) [49, 67]. Enhancers, non-coding and long-range cisregulatory elements, regulate gene transcription by interacting with gene promoters but independently of their position and orientation with respect to the transcription start site [68]. These regulatory elements, marked by monomethylation of histone $\mathrm{H} 3$ at lysine 4 and acetylation of histone $\mathrm{H} 3$ at lysine 27, bind to different proteins involved in the establishment and maintenance of chromatin loopings [68]. Most enhancer elements are normally inaccessible due to closed nucleosome structure, while a subset of sequence-specific transcription factors can act as pioneer factors and erase this barrier to occupy their specific sequences [68]. These pioneer factors make the underlying 
DNA more accessible to the transcriptional machinery, thereby promoting the recruitment of a Mediator complex, and facilitating enhancer interaction with the basal transcription machinery and RNA polymerase II at promoters in a gene-specific manner. Moreover, there exist superenhancers, a small set of enhancers spanning large regions of the genome in a clustered manner, and in cancer cells, super-enhancers are frequently associated with oncogene activation [68].

\section{The TERT gene rearrangements}

Zhao et al. [69] first noticed the rearrangement of the TERT locus coupled with TERT induction/telomerase activation in immortalized human fibroblasts. Then the analyses of mantle cell lymphoma (MCL) using multi-color FISH unraveled the TERT rearrangement in 4/8 MCL-derived cell lines and $1 / 23$ patients [70]. Importantly, all these cell lines and patient cells expressed significantly higher levels of the TERT gene [70]. However, a detailed analysis was not performed in these earlier reports. Recently, using highthroughput sequencing, this genomic event has been identified to activate telomerase in many cancer types, including neuroblastoma, glioblastoma, meningiomas, malignant melanoma and pheochromocytomas, chromophobe renal cell carcinoma (ChRCC), HCC, and among others [71-78] (Fig. 3b). The TCGA cohort patient analyses showed that the structural variants/rearrangements involved in the TERT locus were similarly observed and the highest frequency occurred in sarcoma (22\%) [49] (Fig. 3b).

Based on the analyses of NIH Epigenomics Roadmap data (containing 127 human tissues), Valentijn et al. [73] documented that the 20-kb region upstream of TERT was highly repressed across all investigated tissues and displayed chromatin modifications characteristic of a Polycomb signature, whereas super-enhancers were readily found beyond this repressive region. Thus, the disruption of this Polycomb-silenced region likely leads to TERT activation [73]. TERT rearrangements are not random events, because the majority of the TERT rearrangements lead to the direct overlapping between super-enhancers and juxtaposed TERT coding sequence. Such an overlapping causes enhancer-hijacking through which massive chromatin remodeling and transcriptional activation of the TERT gene is achieved [73]. The TCGA data analyses and many other observations indeed show that TERT rearrangements induce TERT expression much more efficiently than TERT promoter mutations [49].

The TERT rearrangement has been most comprehensively dissected in neuroblasoma, a malignancy of the sympathetic nervous system in infants and young children [71-73]. Peifer et al. [71] observed the recurrent genomic rearrangements in a $50 \mathrm{~kb}$ region proximal of the TERT gene in high risk/stage (III and IV) tumors with a frequency of 23\% (39/169) (Fig. 3a). Similar scenarios were also documented in two other publications [72, 73]. Interestingly, the rearrangement event is mutually exclusive with MYCN aberrations and ATRX mutations [71-73]. MYCN is known to activate the TERT gene and the MYCN amplification is the most frequent genomic alteration in neuroblastoma. ATRX encodes a SWI/SNF chromatinremodeling ATP-dependent helicase, and its mutation leads to the functional inactivation of the gene product, thereby promoting alternative lengthening of telomeres (ALT), a recombination-based mechanism for telomere maintenance [71-73]. Taken together, the different subgroups of neuroblastoma employ different mechanisms for telomere lengthening and sustained cellular proliferation.

\section{Onco-viral DNA insertions}

Infection of oncogenic virus is responsible for up to $15 \%$ of human malignancies [67], and activation of telomerase is one of the key mechanisms behind viral carcinogenesis [47]. It has long been suggested that viral proteins derived from HPV, HBV, EBV, CMV, and others serve as cofactors to activate TERT transcription, as described above [47]. Recent evidence has also accumulated that the integration of the viral DNA into the TERT locus represents additional novel mechanisms for TERT regulation (Fig. 3a).

HBV infection is intimately associated with HCC development, which provides an ideal study model. The integration of the HBV DNA into host genome has long been realized as a pivotal carcinogenic driving-force, however, underlying mechanisms are elusive. In a study of HCC cell lines, the HBV enhancer-containing DNA fragment was found to insert into the 5 ' regulatory region at the $1.6 \mathrm{~kb}$ upstream of the TERT transcription start site and this exogenous viral enhancer drove endogenous TERT transcription in HCC cells [79]. Using a PCR-based assay, Paterlini-Bréchot et al. showed that HBV targeted the TERT gene for integration in 2 out $22 \mathrm{HCC}$ tumors [43]. To thoroughly elucidate the role for HBV-host genome interaction in HCC pathogenesis, Sung et al. [80] conducted a massive sequencing analysis of $81 \mathrm{HBV}$-positive HCC tumors. They found that the TERT locus was the most frequent target as HBV integration breakpoints and the insertion of HBV DNA were identified in 18 of these HCC tumors [80]. Most of the integration breakpoints were located in the TERT promoter region, and almost all the integrations contained at least one viral gene enhancer or promoter [67] (Fig. 3a). Consistently, higher levels of TERT expression were readily seen in these tumors [67, 80]. For HBV-negative HCC, adeno-associated virus type 2 (AAV2), a member of the parvovirus group, may be involved in the oncogenic process. Nault et al. [81] showed 
that AAV2 DNA was integrated into the host genome in 11 of 193 HCC tumors and all the breakpoints affected cancerrelated genes including TERT. The $208 \mathrm{bp}$ AAV2 DNA fragment, cloned from the patient tumor and when inserted into the TERT promoter reporter construct, significantly increased luciferase activity, suggesting a functional significance of the inserted AAV2 sequence in telomerase activation [81].

The findings described above demonstrate that HCC cells acquire TERT overexpression and telomerase activation by hijacking integrated HBV promoters or enhancers at the TERT locus, however, little has been known about the genomic interaction between the host TERT and other oncoviruses. Chen et al. screened for the DNA integration from a panel of onco-viruses including HPV, EBV, and BKV in different types of tumors, and they did detect the presence of insertion events, but integration breakpoints were located at other cancer-driving genes rather than the TERT locus [67]. However, another recent study showed that HPV DNA targeted the TERT locus for integration in cells and tumors derived from head and neck cancer [82], but more detailed and comprehensive analyses are required.

\section{TERT amplification: matter of gene dosages}

The amplification of genes encoding oncogenic products frequently occurs in cancer. For example, the $M Y C N$ amplification is the most common genomic aberration in high-risk neuroblastoma, which leads to MYCN overexpression, driving aggressive diseases [71]. Our earlier study demonstrated that TERT expression was gene-dosage dependent and haploinsufficient for telomere maintenance in human cells [83], suggesting this gene as an amplified target in carcinogenesis. Indeed, we and Keith's group first identified the TERT amplification in human cancer almost 20 years ago $[84,85]$. In neuroblastoma cells, we observed that the TERT gene was typically amplified in doubleminuses, and each cell harbored more than 100 TERT copies [84]. In other tumors, focal copy gains or amplification at the TERT locus were frequently detected $[31,60,86]$. Numerous studies have since then shown the widespread of this genomic event in many different types of malignancies. Moreover, high-throughput next-generation sequencing has been performed on most human cancer types in the last decade, which have provided rich tumor genomic information. Barthel et al. [49] analyzed the TERT gene amplification in the TCGA cohort including 6835 patients and covering 31 tumor types, and the findings are summarized as follow: (i) A total of $4 \%$ the examined tumors exhibit a TERT gene amplification, with high frequency in ovarian cancer, adrenocortical carcinoma, esophageal cancer, lung adenocarcinoma, and squamous carcinoma (Fig. 3c); (ii) TERT amplification is observed in
$3 \%$ of TERT-expressing tumors; and (iii) The highest telomerase activity is found in tumors with a TERT amplification. These results obtained from the TCGA cohort provide a general amplification profile of the TERT gene in human cancer, and indicate a key role of this genomic aberration in activation of telomerase during cancer development and/or progression.

\section{TERT transcription via telomere position effect over long distances (TPE-OLD): genomic-epigenetic interaction}

Genes near telomeres are regulated by a mechanism that depends both on telomere length and on the distance to the gene, so-called telomere position effect (TPE) [5]. More recently, TERT and some other genes, although localized at positions with a certain distance from telomeres (Figs. 2a and $3 \mathrm{a}$ ), were also found to be regulated by telomere length, a TPE-like mechanism or TPE-over long distances (TPEOLD) [22]. In normal young human cells with long telomeres, a telomere-loop structure is formed in the region near the TERT locus, leading to a repressed TERT epigenetic state, however, shortened telomeres in aged cells disrupt the repressive loop, which consequently opens the closed chromatin structure and induces TERT transcription. Because very short telomeres are the most frequent genomic alteration in human tumors, TPE-OLD likely plays a part in TERT/telomerase activation in cancer cells. It is currently unclear whether or how much this mechanism contributes to cancer-related TERT transcription, and whether it cooperates with other regulatory cascades to activate telomerase. The elucidation of these issues will gain insights into not only telomere biology but also the relationship between age and cancer.

\section{Clinical implications/applications in precision oncology}

\section{Biomarkers for cancer diagnosis and disease surveillance}

Cancer-specific TERT expression and telomerase activation has always aroused great enthusiasm for a potential clinical application of TERT/telomerase-based assays in the cancer field. However, a number of problems (such as unstable TERT mRNA and enzymatic activity) impede reliable utility of a direct TERT expression or telomerase activity assay for cancer diagnostic or monitoring purpose, whereas widespread TERT promoter mutations in different tumors pave new avenues [31]. The non-invasive detection of a mutant TERT promoter is especially attractive and has been evaluated in plasma, urine and cerebrospinal fluid (CSF) for the diagnosis/monitoring of HCC, bladder cancer and glioblastoma, respectively [31, 87-90]. These proof-of- 
concept studies have shown usefulness of the mutant TERT promoter as a non-invasive assay biomarker for these malignancies.

Since many types of human cancer lack any TERT promoter mutations, and even in bladder cancer and glioblastoma, up to $30 \%$ of the tumors are negative for a mutation, other telomerase-related markers are apparently required for those patients. The hypermethylated TERT promoter has also been shown to be unique to human cancer as described above, and might serve as a diagnostic biomarker. We have recently identified two methylated CpGs in the TERT promoter region specific to tumors from patients with gastrointestinal cancer (GIC), and the methylated sites were detectable in stool from GIC patients, with sensitivity and specificity comparable to a fecal occult blood test [24]. Bougel et al. found that the methylated TERT promoter detection in CSF could predict leptomeningeal metastasis [91]. These studies suggest the feasibility of TERT promoter methylation analyses as an additional tool in noninvasive cancer diagnosis and disease surveillance.

\section{Outcome prediction}

Numerous clinical studies have evaluated telomerase/ TERT-related alterations as prognostic factors for cancer patients. Higher TERT expression in tumors was observed to predict poor patient outcomes in a panel of cancer types [31, 49, 92]. In neuroblastoma, tumors without detectable TERT mRNA frequently undergo spontaneous regression [93], whereas TERT expression, and/or its gene amplification or rearrangements are closely associated with a highrisk/aggressive disease and shorter survival [71-73]. The presence of TERT promoter mutations has recently been shown as a unfavorable prognostic factor in a number of cancer types including papillary thyroid carcinoma, glioblastoma, bladder cancer, and others [31, 94-97]. In addition, the association between TERT promoter hypermehtylation and poor outcomes or progression was reported in brain tumors and adrenocortical carcinoma [25, 98]. Collectively, the aberrant TERT expression and TERT-related genomic alterations may serve as prognostic factors in multiple types of cancer, and the further evaluation including large cohorts of patients is required for future clinical application.

\section{Concluding remarks}

Homo sapiens have acquired robust protective mechanisms against cancer by repressing telomerase coupled with shorter telomeres over a long evolution period. Erasing this natural barrier is required for malignant transformation of human somatic cells and in most cases, is achieved by the TERT gene de-repression/telomerase reactivation. Thus, a mechanistic elucidation underlying cancer-specific activation of the TERT transcription is of intense interest to cancer research. During the last 20 years, this issue has been extensively studied. We learnt much from the cloning of the TERT gene and its promoter, and identification of its key transcription factors (both positive and negative), however, the findings made in the past 6 years have significantly contributed to our in-depth understanding of telomerase biology in cancer, and the genomic aberrations have started to take center stage: the hotspot mutation at 5' regulatory promoter region and rearrangements at the TERT locus are emerging as new players in activating TERT transcription. It should be pointed out that these genomic aberrations stimulate TERT expression by triggering massive epigenetic alterations and disrupting repressive chromatins locally. Therefore, a close genomic-epigenetic interaction is required for telomerase activation, like TPE-OLD-mediated TERT transcription. Interestingly, TERT promoter methylation is required for cancer cells to activate TERT transcription, while TERT induction in turn promotes the aberrant methylation by upregulating the expression of DNA methyltransferases, which forms a positive feedback loop [20, 23].

These new findings not only lead to profound mechanistic understanding of the cancer-specific TERT transcription, but also provide useful tools for clinical managements of cancer patients. However, a key challenge is how to translate these findings into a telomerase-based therapeutic strategy. The ETS family member GABPB1, required for the TERT transcription in TERT promoter-bearing glioblastoma cells, has been proposed as a target for glioblastoma treatment [56], but our results showed that inhibiting GABPA or GABPB1 robustly increased invasiveness of TC cells despite significant downregulation of TERT expression [59]. Likely, the GABPA/B function is context-dependent. Further studies are required for the rational development of TERT-based cancer therapeutic interventions.

Acknowledgements This work was supported by grants from China Postdoctoral Science Foundation Grant (2019M652404), the Swedish Cancer Society, Swedish Research Council, the Cancer Society in Stockholm, Karolinska Institutet Foundation, Swedish Foundation for International Cooperation in Research and Higher Education (STINT), Ruth and Richard Julin Foundation and Nanna Svartź Foundation.

\section{Compliance with ethical standards}

Conflict of interest The authors declare that they have no conflict of interest.

Publisher's note: Springer Nature remains neutral with regard to jurisdictional claims in published maps and institutional affiliations. 
Open Access This article is licensed under a Creative Commons Attribution 4.0 International License, which permits use, sharing, adaptation, distribution and reproduction in any medium or format, as long as you give appropriate credit to the original author(s) and the source, provide a link to the Creative Commons license, and indicate if changes were made. The images or other third party material in this article are included in the article's Creative Commons license, unless indicated otherwise in a credit line to the material. If material is not included in the article's Creative Commons license and your intended use is not permitted by statutory regulation or exceeds the permitted use, you will need to obtain permission directly from the copyright holder. To view a copy of this license, visit http://creativecommons. org/licenses/by/4.0/.

\section{References}

1. Vogelstein B, Kinzler KW. The path to cancer-three strikes and you're out. N Engl J Med. 2015;373:1895-8.

2. Seluanov A, Gladyshev VN, Vijg J, Gorbunova V. Mechanisms of cancer resistance in long-lived mammals. Nat Rev Cancer. 2018;18:433-41.

3. Gomes NM, Ryder OA, Houck ML, Charter SJ, Walker W, Forsyth NR, et al. Comparative biology of mammalian telomeres: hypotheses on ancestral states and the roles of telomeres in longevity determination. Aging Cell. 2011;10:761-8.

4. Hahn WC, Counter CM, Lundberg AS, Beijersbergen RL, Brooks MW, Weinberg RA. Creation of human tumour cells with defined genetic elements. Nature. 1999;400:464-8.

5. Shay JW, Wright WE. Telomeres and telomerase: three decades of progress. Nat Rev Genet. 2019;20:299-309.

6. Hanahan D, Weinberg RA. Hallmarks of cancer: the next generation. Cell. 2011;144:646-74.

7. Bodnar AG, Ouellette M, Frolkis M, Holt SE, Chiu CP, Morin $\mathrm{GB}$, et al. Extension of life-span by introduction of telomerase into normal human cells. Science. 1998;279:349-52.

8. Im E, Yoon JB, Lee HW, Chung KC. Human telomerase reverse transcriptase (hTERT) positively regulates $26 \mathrm{~S}$ proteasome activity. J Cell Physiol. 2017;232:2083-93.

9. Hu C, Ni Z, Li BS, Yong X, Yang X, Zhang JW, et al. hTERT promotes the invasion of gastric cancer cells by enhancing FOXO3a ubiquitination and subsequent ITGB1 upregulation. Gut. 2017;66:31-42.

10. Saretzki G. Extra-telomeric functions of human telomerase: cancer, mitochondria and oxidative stress. Curr Pharm Des. 2014;20:6386-403.

11. Masutomi K, Possemato R, Wong JM, Currier JL, Tothova Z, Manola JB, et al. The telomerase reverse transcriptase regulates chromatin state and DNA damage responses. Proc Natl Acad Sci USA. 2005;102:8222-7.

12. Liu Z, Li Q, Li K, Chen L, Li W, Hou M, et al. Telomerase reverse transcriptase promotes epithelial-mesenchymal transition and stem cell-like traits in cancer cells. Oncogene. 2013;32:4203-13.

13. Zhang K, Guo Y, Wang X, Zhao H, Ji Z, Cheng C, et al. WNT/ beta-catenin directs self-renewal symmetric cell division of hTERT(high) prostate cancer stem cells. Cancer Res. 2017;77:2534-47.

14. Ding D, Xi P, Zhou J, Wang M, Cong YS. Human telomerase reverse transcriptase regulates MMP expression independently of telomerase activity via NF-kappaB-dependent transcription. FASEB J. 2013;27:4375-83.

15. Lassmann T, Maida Y, Tomaru Y, Yasukawa M, Ando Y, Kojima $\mathrm{M}$, et al. Telomerase reverse transcriptase regulates microRNAs. Int J Mol Sci. 2015;16:1192-208.
16. Drevytska TI, Nagibin VS, Gurianova VL, Kedlyan VR, Moibenko AA, Dosenko VE. Silencing of TERT decreases levels of miR-1, miR-21, miR-29a and miR-208a in cardiomyocytes. Cell Biochem Funct. 2014;32:565-70.

17. Park JI, Venteicher AS, Hong JY, Choi J, Jun S, Shkreli M, et al. Telomerase modulates Wnt signalling by association with target gene chromatin. Nature. 2009;460:66-72.

18. Maida Y, Masutomi K. Telomerase reverse transcriptase moonlights: therapeutic targets beyond telomerase. Cancer Sci. 2015;106:1486-92.

19. Liu N, Ding D, Hao W, Yang F, Wu X, Wang M, et al. hTERT promotes tumor angiogenesis by activating VEGF via interactions with the Sp1 transcription factor. Nucleic Acids Res. 2016;44:8693-703.

20. Yu J, Yuan X, Sjoholm L, Liu T, Kong F, Ekstrom TJ, et al. Telomerase reverse transcriptase regulates DNMT3B expression/ aberrant DNA methylation phenotype and AKT activation in hepatocellular carcinoma. Cancer Lett. 2018;434:33-41.

21. Cong YS, Wen J, Bacchetti S. The human telomerase catalytic subunit hTERT: organization of the gene and characterization of the promoter. Hum Mol Genet. 1999;8:137-42.

22. Kim W, Ludlow AT, Min J, Robin JD, Stadler G, Mender I, et al. Regulation of the human telomerase gene TERT by telomere position effect-over long distances (TPE-OLD): implications for aging and cancer. PLoS Biol. 2016;14:e2000016.

23. Lee DD, Leao R, Komosa M, Gallo M, Zhang CH, Lipman T, et al. DNA hypermethylation within TERT promoter upregulates TERT expression in cancer. J Clin Invest. 2019;129:223-9.

24. Liu L, Liu C, Fotouhi O, Fan Y, Wang K, Xia C, et al. TERT promoter hypermethylation in gastrointestinal cancer: a potential stool biomarker. Oncologist. 2017;22:1178-88.

25. Svahn F, Juhlin CC, Paulsson JO, Fotouhi O, Zedenius J, Larsson $\mathrm{C}$, et al. Telomerase reverse transcriptase promoter hypermethylation is associated with metastatic disease in abdominal paraganglioma. Clin Endocrinol. 2018;88:343-5.

26. Wang N, Xu D, Sofiadis A, Hoog A, Vukojevic V, Backdahl M, et al. Telomerase-dependent and independent telomere maintenance and its clinical implications in medullary thyroid carcinoma. J Clin Endocrinol Metab. 2014;99:E1571-9.

27. Takakura M, Kyo S, Kanaya T, Hirano H, Takeda J, Yutsudo M, et al. Cloning of human telomerase catalytic subunit (hTERT) gene promoter and identification of proximal core promoter sequences essential for transcriptional activation in immortalized and cancer cells. Cancer Res. 1999;59:551-7.

28. Wong MS, Wright WE, Shay JW. Alternative splicing regulation of telomerase: a new paradigm? Trends Genet. 2014;30:430-8.

29. Li W, Li L, Liu Z, Liu C, Liu Z, Straat K, et al. Expression of the full-length telomerase reverse transcriptase (hTERT) transcript in both malignant and normal gastric tissues. Cancer Lett. 2008;260:28-36.

30. Fan Y, Liu Z, Fang X, Ge Z, Ge N, Jia Y, et al. Differential expression of full-length telomerase reverse transcriptase mRNA and telomerase activity between normal and malignant renal tissues. Clin Cancer Res. 2005;11:4331-7.

31. Liu T, Yuan X, Xu D. Cancer-specific telomerase reverse transcriptase (TERT) promoter mutations: biological and clinical implications. Genes. 2016;7:E38. pii

32. Xu D, Popov N, Hou M, Wang Q, Bjorkholm M, Gruber A, et al. Switch from Myc/Max to Mad1/Max binding and decrease in histone acetylation at the telomerase reverse transcriptase promoter during differentiation of HL60 cells. Proc Natl Acad Sci USA. 2001;98:3826-31.

33. Casillas MA, Brotherton SL, Andrews LG, Ruppert JM, Tollefsbol TO. Induction of endogenous telomerase (hTERT) by c-Myc in WI-38 fibroblasts transformed with specific genetic elements. Gene. 2003;316:57-65. 
34. Wang J, Xie LY, Allan S, Beach D, Hannon GJ. Myc activates telomerase. Genes Dev. 1998;12:1769-74.

35. Cassar L, Li H, Pinto AR, Nicholls C, Bayne S, Liu JP. Bone morphogenetic protein-7 inhibits telomerase activity, telomere maintenance, and cervical tumor growth. Cancer Res. 2008;68:9157-66.

36. Gewin L, Myers H, Kiyono T, Galloway DA. Identification of a novel telomerase repressor that interacts with the human papillomavirus type-16 E6/E6-AP complex. Genes Dev. 2004;18:2269-82.

37. Koshiji M, Kageyama Y, Pete EA, Horikawa I, Barrett JC, Huang LE. HIF-1alpha induces cell cycle arrest by functionally counteracting Myc. Embo J. 2004;23:1949-56.

38. Li Y, Liu L, Tollefsbol TO. Glucose restriction can extend normal cell lifespan and impair precancerous cell growth through epigenetic control of hTERT and p16 expression. FASEB J. 2010;24:1442-53.

39. Zeng J, Wang L, Li Q, Li W, Bjorkholm M, Jia J, et al. FoxM1 is up-regulated in gastric cancer and its inhibition leads to cellular senescence, partially dependent on p27 kip1. J Pathol. 2009;218:419-27.

40. Xu D, Dwyer J, Li H, Duan W, Liu JP. Ets2 maintains hTERT gene expression and breast cancer cell proliferation by interacting with c-Myc. J Biol Chem. 2008;283:23567-80.

41. Chou WC, Hawkins AL, Barrett JF, Griffin CA, Dang CV. Arsenic inhibition of telomerase transcription leads to genetic instability. J Clin Invest. 2001;108:1541-7.

42. Takakura M, Kyo S, Sowa Y, Wang Z, Yatabe N, Maida Y, et al. Telomerase activation by histone deacetylase inhibitor in normal cells. Nucleic Acids Res. 2001;29:3006-11.

43. Paterlini-Brechot P, Saigo K, Murakami Y, Chami M, Gozuacik D, Mugnier C, et al. Hepatitis B virus-related insertional mutagenesis occurs frequently in human liver cancers and recurrently targets human telomerase gene. Oncogene. 2003;22:3911-6.

44. Zhou J, Mao B, Zhou Q, Ding D, Wang M, Guo P, et al. Endoplasmic reticulum stress activates telomerase. Aging Cell. 2014;13:197-200.

45. Liu C, Fang X, Ge Z, Jalink M, Kyo S, Bjorkholm M, et al. The telomerase reverse transcriptase (hTERT) gene is a direct target of the histone methyltransferase SMYD3. Cancer Res. 2007;67:2626-31.

46. Ge Z, Liu C, Bjorkholm M, Gruber A, Xu D. Mitogen-activated protein kinase cascade-mediated histone $\mathrm{H} 3$ phosphorylation is critical for telomerase reverse transcriptase expression/telomerase activation induced by proliferation. Mol Cell Biol. 2006;26:230-7.

47. Bellon M, Nicot C. Regulation of telomerase and telomeres: human tumor viruses take control. J Natl Cancer Inst. 2008;100:98-108.

48. Straat K, Liu C, Rahbar A, Zhu Q, Liu L, Wolmer-Solberg N, et al. Activation of telomerase by human cytomegalovirus. J Natl Cancer Inst. 2009;101:488-97.

49. Barthel FP, Wei W, Tang M, Martinez-Ledesma E, Hu X, Amin $\mathrm{SB}$, et al. Systematic analysis of telomere length and somatic alterations in 31 cancer types. Nat Genet. 2017;49:349-57.

50. Horn S, Figl A, Rachakonda PS, Fischer C, Sucker A, Gast A, et al. TERT promoter mutations in familial and sporadic melanoma. Science. 2013;339:959-61.

51. Huang FW, Hodis E, Xu MJ, Kryukov GV, Chin L, Garraway LA. Highly recurrent TERT promoter mutations in human melanoma. Science. 2013;339:957-9.

52. Bell RJ, Rube HT, Xavier-Magalhaes A, Costa BM, Mancini A, Song JS. Understanding TERT Promoter Mutations: A Common Path to Immortality. Mol Cancer Res. 2016;14:315-23.

53. Chiba K, Johnson JZ, Vogan JM, Costa BM, Mancini A, Song JS. Cancer-associated TERT promoter mutations abrogate telomerase silencing. Elife 2015;4. https://doi.org/10.7554/eLife.07918.

54. Li C, Wu S, Wang H, Bi X, Yang Z, Du Y, et al. The C228T mutation of TERT promoter frequently occurs in bladder cancer stem cells and contributes to tumorigenesis of bladder cancer. Oncotarget. 2015;6:19542-51.

55. Bell RJ, Rube HT, Kreig A, Mancini A, Fouse SD, Nagarajan RP, et al. The transcription factor GABP selectively binds and activates the mutant TERT promoter in cancer. Science. 2015;348:1036-9.

56. Mancini A, Xavier-Magalhaes A, Woods WS, Nguyen KT, Amen AM, Hayes JL, et al. Disruption of the beta1L isoform of GABP reverses glioblastoma replicative immortality in a TERT promoter mutation-dependent manner. Cancer Cell. 2018;34:513-28 e518.

57. Stern JL, Theodorescu D, Vogelstein B, Papadopoulos N, Cech TR. Mutation of the TERT promoter, switch to active chromatin, and monoallelic TERT expression in multiple cancers. Genes Dev. 2015;29:2219-24

58. Sizemore GM, Pitarresi JR, Balakrishnan S, Ostrowski MC. The ETS family of oncogenic transcription factors in solid tumours. Nat Rev Cancer. 2017;17:337-51.

59. Yuan X, Mu N, Wang N, Straat K, Sofiadis A, Guo Y, et al. GABPA inhibits invasion/metastasis in papillary thyroid carcinoma by regulating DICER1 expression. Oncogene. 2019;38:965-79.

60. Xie H, Liu T, Wang N, Bjornhagen V, Hoog A, Larsson C, et al. TERT promoter mutations and gene amplification: promoting TERT expression in Merkel cell carcinoma. Oncotarget. 2014;5:10048-57.

61. Mitchell TJ, Turajlic S, Rowan A, Nicol D, Farmery JHR, O'Brien $\mathrm{T}$, et al. Timing the landmark events in the evolution of clear cell renal cell cancer: TRACERx renal. Cell. 2018;173:611-23 e617.

62. Liu T, Wang N, Cao J, Sofiadis A, Dinets A, Zedenius J, et al. The age- and shorter telomere-dependent TERT promoter mutation in follicular thyroid cell-derived carcinomas. Oncogene. 2014;33:4978-84.

63. Chiba K, Lorbeer FK, Shain AH, McSwiggen DT, Schruf E, Oh A, et al. Mutations in the promoter of the telomerase gene TERT contribute to tumorigenesis by a two-step mechanism. Science. 2017;357:1416-20.

64. Maryoung L, Yue Y, Young A, Newton CA, Barba C, van Oers NS, et al. Somatic mutations in telomerase promoter counterbalance germline loss-of-function mutations. J Clin Invest. 2017;127:982-6.

65. Yuan X, Cheng G, Yu J, Zheng S, Sun C, Sun Q, et al. The TERT promoter mutation incidence is modified by germline TERT rs2736098 and rs2736100 polymorphisms in hepatocellular carcinoma. Oncotarget. 2017;8:23120-9.

66. Dahlstrom J, Liu T, Yuan X, Saft L, Ghaderi M, Wei YB, et al. TERT rs2736100 genotypes are associated with differential risk of myeloproliferative neoplasms in Swedish and Chinese male patient populations. Ann Hematol. 2016;95:1825-32.

67. Chen X, Kost J, Sulovari A, Wong N, Liang W, Cao J, et al. A virome-wide clonal integration analysis platform for discovering cancer viral etiology. Genome Res. 2019;29:819-30.

68. Levine M, Cattoglio C, Tjian R. Looping back to leap forward: transcription enters a new era. Cell. 2014;157:13-25.

69. Zhao Y, Wang S, Popova EY, Grigoryev SA, Zhu J. Rearrangement of upstream sequences of the hTERT gene during cellular immortalization. Genes Chromosomes Cancer. 2009;48:963-74.

70. Schilling G, Penas EM, Janjetovic S, Oliveira-Ferrer L, Braig M, Behrmann $\mathrm{P}$, et al. Molecular characterization of chromosomal band 5p15.33: a recurrent breakpoint region in mantle cell lymphoma involving the TERT-CLPTM1L locus. Leuk Res. 2013;37:280-6.

71. Peifer M, Hertwig F, Roels F, Dreidax D, Gartlgruber M, Menon $\mathrm{R}$, et al. Telomerase activation by genomic rearrangements in high-risk neuroblastoma. Nature. 2015;526:700-4. 
72. Ackermann S, Cartolano M, Hero B, Welte A, Kahlert Y, Roderwieser A, et al. A mechanistic classification of clinical phenotypes in neuroblastoma. Science. 2018;362:1165-70.

73. Valentijn LJ, Koster J, Zwijnenburg DA, Hasselt NE, van Sluis P, Volckmann R, et al. TERT rearrangements are frequent in neuroblastoma and identify aggressive tumors. Nat Genet. 2015;47:1411-4.

74. Davis CF, Ricketts CJ, Wang M, Yang L, Cherniack AD, Shen H, et al. The somatic genomic landscape of chromophobe renal cell carcinoma. Cancer Cell. 2014;26:319-30.

75. Bayard Q, Meunier L, Peneau C, Renault V, Shinde J, Nault JC, et al. Cyclin A2/E1 activation defines a hepatocellular carcinoma subclass with a rearrangement signature of replication stress. Nat Commun. 2018;9:5235.

76. Diplas BH, He X, Brosnan-Cashman JA, Liu H, Chen LH, Wang $\mathrm{Z}$, et al. The genomic landscape of TERT promoter wildtype-IDH wildtype glioblastoma. Nat Commun. 2018;9:2087.

77. Dwight T, Flynn A, Amarasinghe K, Benn DE, Lupat R, Li J, et al. TERT structural rearrangements in metastatic pheochromocytomas. Endocr Relat Cancer. 2018;25:1-9.

78. Juratli TA, McCabe D, Nayyar N, Williams EA, Silverman IM, Tummala SS, et al. DMD genomic deletions characterize a subset of progressive/higher-grade meningiomas with poor outcome. Acta Neuropathol. 2018;136:779-92.

79. Horikawa I, Barrett JC. cis-Activation of the human telomerase gene (hTERT) by the hepatitis B virus genome. J Natl Cancer Inst. 2001;93:1171-3.

80. Sung WK, Zheng H, Li S, Chen R, Liu X, Li Y, et al. Genomewide survey of recurrent $\mathrm{HBV}$ integration in hepatocellular carcinoma. Nat Genet. 2012;44:765-9.

81. Nault JC, Datta S, Imbeaud S, Franconi A, Mallet M, Couchy G, et al. Recurrent AAV2-related insertional mutagenesis in human hepatocellular carcinomas. Nat Genet. 2015;47:1187-93.

82. Chang KP, Wang CI, Pickering CR, Huang Y, Tsai CN, Tsang $\mathrm{NM}$, et al. Prevalence of promoter mutations in the TERT gene in oral cavity squamous cell carcinoma. Head Neck. 2017;39:1131-7.

83. Zhang A, Zheng C, Hou M, Lindvall C, Li KJ, Erlandsson F, et al. Deletion of the telomerase reverse transcriptase gene and haploinsufficiency of telomere maintenance in Cri du chat syndrome. Am J Hum Genet. 2003;72:940-8.

84. Zhang A, Zheng C, Lindvall C, Hou M, Ekedahl J, Lewensohn R, et al. Frequent amplification of the telomerase reverse transcriptase gene in human tumors. Cancer Res. 2000;60:6230-5.

85. Zhao JQ, Glasspool RM, Hoare SF, Bilsland A, Szatmari I, Keith WN. Activation of telomerase rna gene promoter activity by NF$\mathrm{Y}, \mathrm{Sp} 1$, and the retinoblastoma protein and repression by $\mathrm{Sp} 3$. Neoplasia. 2000;2:531-9.
86. Lin Y, Seger N, Tsagkozis P, Hesla AC, Ghaderi M, Chen Y, et al. Telomerase promoter mutations and copy number alterations in solitary fibrous tumours. J Clin Pathol. 2018;71:832-9.

87. Labgaa I, Villacorta-Martin C, D'Avola D, Craig AJ, von Felden J, Martins-Filho SN, et al. A pilot study of ultra-deep targeted sequencing of plasma DNA identifies driver mutations in hepatocellular carcinoma. Oncogene. 2018;37:3740-52.

88. Hurst CD, Platt FM, Knowles MA. Comprehensive mutation analysis of the TERT promoter in bladder cancer and detection of mutations in voided urine. Eur Urol. 2014;65:367-8.

89. Wang K, Liu T, Liu C, Meng Y, Yuan X, Liu L, et al. TERT promoter mutations and TERT mRNA but not FGFR3 mutations are urinary biomarkers in Han Chinese patients with urothelial bladder cancer. Oncologist. 2015;20:263-9.

90. Juratli TA, Stasik S, Zolal A, Schuster C, Richter S, Daubner D, et al. TERT promoter mutation detection in cell-free tumorderived DNA in patients with IDH wild-type glioblastomas: a pilot prospective study. Clin Cancer Res. 2018;24:5282-91.

91. Bougel S, Lhermitte B, Gallagher G, de Flaugergues JC, Janzer RC, Benhattar J. Methylation of the hTERT promoter: a novel cancer biomarker for leptomeningeal metastasis detection in cerebrospinal fluids. Clin Cancer Res. 2013;19:2216-23.

92. Ma R, Liu C, Lu M, Yuan X, Cheng G, Kong F, et al. The TERT locus genotypes of rs2736100-CC/CA and rs2736098-AA predict shorter survival in renal cell carcinoma. Urol Oncol. 2019;37:301 e301-301 e310.

93. Hiyama E, Hiyama K, Yokoyama T, Matsuura Y, Piatyszek MA, Shay JW. Correlating telomerase activity levels with human neuroblastoma outcomes. Nat Med. 1995;1:249-55.

94. Xing M, Liu R, Liu X, Murugan AK, Zhu G, Zeiger MA, et al. BRAF V600E and TERT promoter mutations cooperatively identify the most aggressive papillary thyroid cancer with highest recurrence. J Clin Oncol. 2015;32:2718-26.

95. Sahm F, Schrimpf D, Olar A, Koelsche C, Reuss D, Bissel J, et al. TERT promoter mutations and risk of recurrence in meningioma. J Natl Cancer Inst. 2015;108: https://doi.org/10.1093/jnci/ djv1377.

96. Brennan CW, Verhaak RG, McKenna A, Campos B, Noushmehr $\mathrm{H}$, Salama SR, et al. The somatic genomic landscape of glioblastoma. Cell. 2016;155:462-77.

97. Wang X, Li X, Xu F, Zhang Y, Liu H, Tao Y. Association of telomerase reverse transcriptase promoter mutations with the prognosis of glioma patients: a meta-analysis. Mol Neurobiol. 2016;53:2726-32.

98. Castelo-Branco P, Choufani S, Mack S, Gallagher D, Zhang C, Lipman $\mathrm{T}$, et al. Methylation of the TERT promoter and risk stratification of childhood brain tumours: an integrative genomic and molecular study. Lancet Oncol. 2013;14:534-42. 